Research Article

\title{
Model for Ray-Based UTD Simulations of the Human Body Shadowing Effect in 5G Wireless Systems
}

\author{
Lukasz Januszkiewicz (iD) \\ Institute of Electronics, Lodz University of Technology, Wólczanska 211/215 Street, 90-924 Łódź, Poland \\ Correspondence should be addressed to Łukasz Januszkiewicz; lukasz.januszkiewicz@gmail.com
}

Received 4 April 2018; Revised 4 July 2018; Accepted 9 July 2018; Published 24 July 2018

Academic Editor: Herve Aubert

Copyright (C) 2018 tukasz Januszkiewicz. This is an open access article distributed under the Creative Commons Attribution License, which permits unrestricted use, distribution, and reproduction in any medium, provided the original work is properly cited.

\begin{abstract}
Shadowing effects caused by the obstructing presence of a human body can result in increased path loss in indoor wireless systems. This paper proposes a simplified model of a human body for use in ray-tracing simulations of indoor wireless communication systems based on the uniform theory of diffraction (UTD). The human body shadowing effect was first investigated using measurements and computer simulations employing the finite-difference time-domain method (FDTD). Based on the results, a human body model was elaborated for use in ray-based Remcom XGtd software. The model was developed for the $3.6 \mathrm{GHz}$ band, which has been allocated for $5 \mathrm{G}$ wireless systems in many countries.
\end{abstract}

\section{Introduction}

Wireless systems used for personal communication utilize a wide range of frequency bands, from $800 \mathrm{MHz}$ to $6 \mathrm{GHz}$. There are important considerations for the designers of wireless systems operating in this range in close proximity to people. For example, the absorption of electromagnetic energy can cause rises in tissue temperature and requires careful monitoring. The absorption of electromagnetic energy can, furthermore, have a significant influence on path loss between the wireless transceivers. The impedance and radiation patterns of the antennas may also be affected by proximity to a human body. Designers of personal cellular communication systems, in which the transmitted power of the portable terminals reaches several watts, have focused mainly on analysis of the specific absorbance rate (SAR) and the influence of the human body on antenna properties. For designers of wireless sensor networks, which utilize low-power miniature transceivers located on the body, path loss is a more important factor.

The body shadowing effect can also impact the performance of wireless systems located at greater distances from people. It becomes a consideration when the distances between the transmitter and the receiver are large enough to include in their geometry the possible presence of humans, such as in the case of off-body transmission in wireless body area networks, where other people in the same area may influence the transmission path. The same effect can also be a factor in wireless systems that operate close to people but do not use on-body transceivers. In such systems, the Line-of-Sight (LOS) components of the radio signal are susceptible to human body shadowing and the received signal fluctuates slowly around a mean value in displacements of hundreds of wavelengths $[1,2]$. Shadowing occurs due to the blocking of LOS components by a human body located between the transmitter and receiver. This effect is presented in Figure 1, where the transmitter and receiver can operate either under LOS conditions (Figure 1(a)) or in the presence of a body that shadows the transmitted signal (Figure 1(b)).

The body shadowing effect has been the subject of extensive studies in recent years, due to the growing popularity of wireless systems designed to work in close proximity to people [1-10]. Different models have been developed, taking into account not only the effect of human body shadowing on time variation, but also other factors in the 

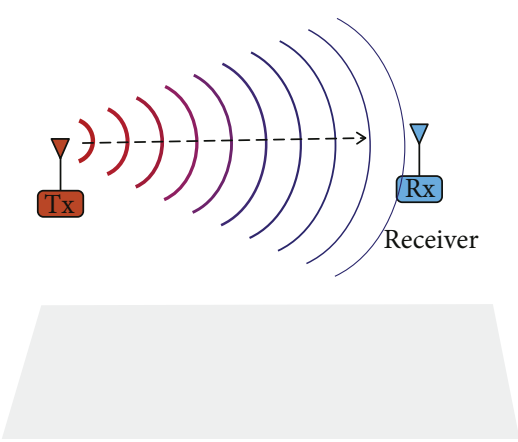

(a)

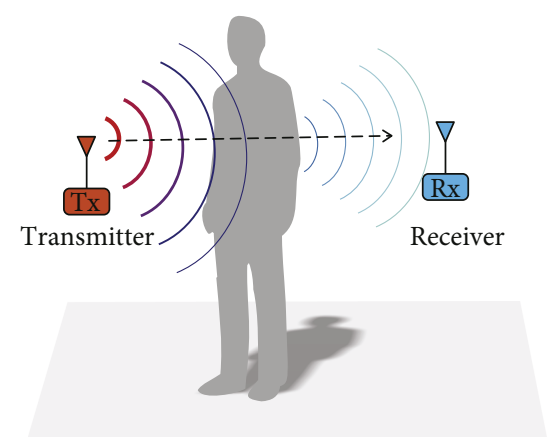

(b)

FIGURE 1: Body shadowing effect: (a) Line-of-Sight case; (b) body shadowing case.

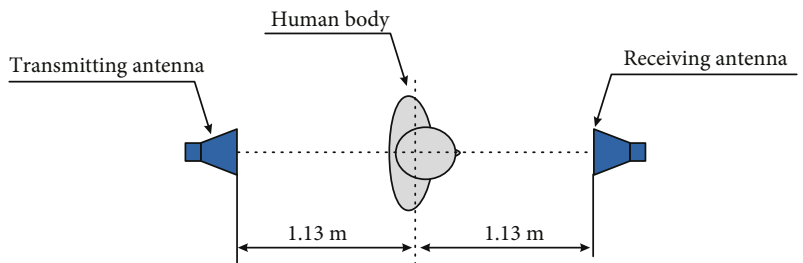

(a)

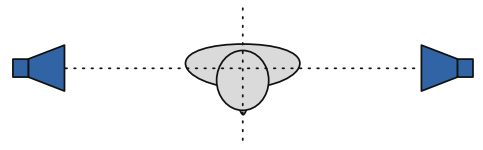

(b)

Figure 2: Geometry of the measurement experiment: (a) body orientation 1 and (b) body orientation 2.

indoor environment which can influence the propagation of the signal [3-5]. A number of studies have applied the experimental approach to measure the path loss introduced by human bodies, for a wide range of frequencies and in a variety of geometrical scenarios [6-8]. The results have then been used to define the parameters of propagation models in a deterministic way $[9,10]$.

The body shadowing effect influences the properties of wireless channels and should be considered in the design stage. The design of wireless networks is now supported by computer programs which simulate the propagation of electromagnetic waves between the transmitters and receivers. These simulations can provide information concerning path loss in a particular propagation environment, which may enable the reliability of the wireless system to be improved dramatically. To simulate wireless systems operating in large areas, such as buildings or streets in built-up areas, ray-based computer programs are used, which employ the concepts of energy transmission, reflection, and diffraction. These can model the effects of electromagnetic wave propagation in very large objects, which would be impossible using Maxwell's equations.

In this paper, a human body model is presented that can be used in a ray-based computer program that utilizes the uniform theory of diffraction (UTD) to model the body shadowing effect. The model was developed for the $3.6 \mathrm{GHz}$ band because this frequency range is being considered for fifth generation (5G) wireless systems in many countries in Europe, including Poland. The Radio Spectrum Policy Group identifies the $3.6 \mathrm{GHz}$ band as the primary band for the introduction of 5G in Europe, even before 2020 [11]. To identify the model parameters, the body shadowing effect was first investigated using measurements and computer simulations employing the finite-difference time-domain method (FDTD). Based on the results, a human body model was elaborated for use in ray-based Remcom XGtd software.

\section{Materials and Methods}

2.1. Measurement of the Body Shadowing Effect. The body shadowing effect was first examined via measurements. The measurement setup was configured according to the schema in Figure 2. The transmitting and receiving antennas were connected to an Anritsu MS4644B vector network analyzer, which measured the transmittance between the two ports (the module of parameter S21 of the scattering matrix). The calibration plane was on the connectors of the antennas, so the influence of the feeding cables was eliminated. Rohde \& Schwarz HF907 measurement antennas were used. These compact broadband double-ridged waveguide horn antennas have a directional radiation pattern and linear polarization. To investigate the influence of a human body on signal transmission, measurements were initially performed in the freespace condition and then with body shadowing. In the latter case, a human subject was positioned between the transmitting and receiving antennas. Measurements were performed for two orientations of the body (see Figure 2). In the first orientation, the front of the human subject faced the receiving antenna. In the second orientation, the left arm of the human subject was on the side of the receiving antenna.

To avoid the influence of walls, measurements were carried out in a small semianechoic chamber. Figure 3 presents the measurement setup. The distance between the antennas was limited by the size of the chamber to $2.26 \mathrm{~m}$, and the 


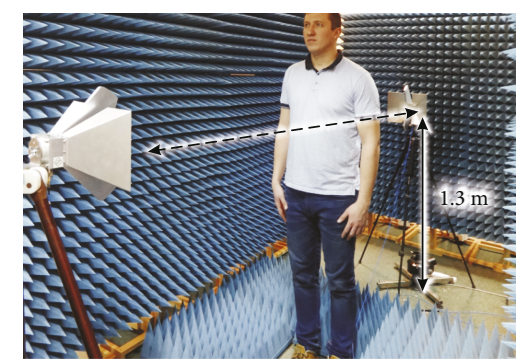

Figure 3: The measurement setup.

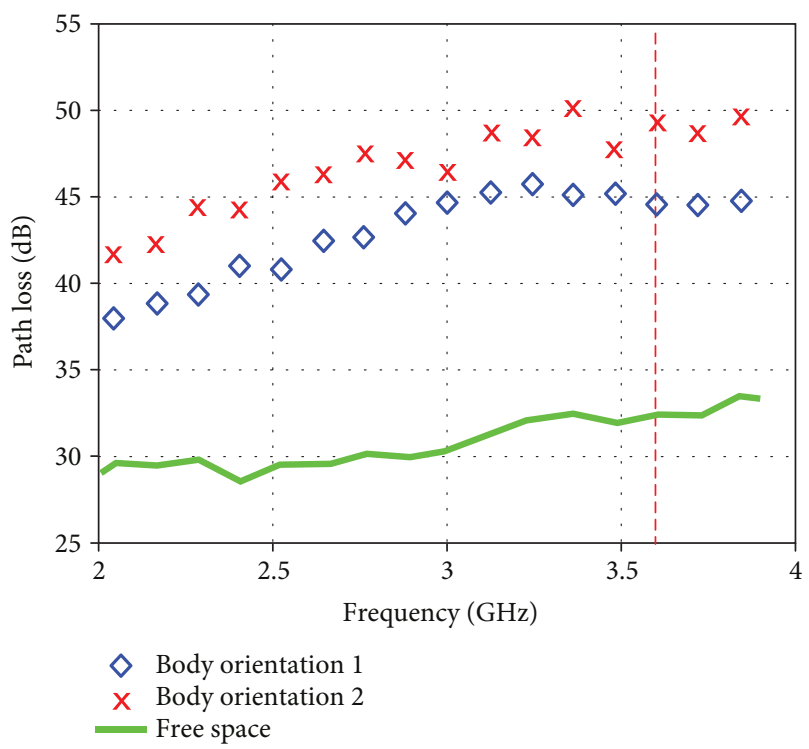

FIGURE 4: Results of body shadowing measurements as a function of frequency. The $3.6 \mathrm{GHz}$ frequency is marked with a red dotted line.

height of the antenna from the ground was $1.3 \mathrm{~m}$ (measured from the electrical centre of the antenna). The height of the human subject was approximately $1.84 \mathrm{~m}$. The human subject was positioned in the middle of the measurement setup.

The results of body shadowing measurements obtained in the frequency domain are presented in Figure 4. The measurements were carried out in the $2 \mathrm{GHz}$ to $4 \mathrm{GHz}$ band. In further investigations, the $3.6 \mathrm{GHz}$ frequency was considered because of its importance for $5 \mathrm{G}$ systems. This band is marked in Figure 4 with a red dotted line. The free-space loss for this particular frequency was $33 \mathrm{~dB}$. This corresponds very well with the theoretical value for free-space path loss that in the considered case is $31.6 \mathrm{~dB}$. The path loss for body orientation 1 was $44 \mathrm{~dB}$, and for orientation 2, path loss was $49 \mathrm{~dB}$. These values were taken as the references for further investigations of the body shadowing effect using computer simulations.

\subsection{Simulations of the Body Shadowing Effect Using the Finite-} Difference Time-Domain Method. The finite-difference timedomain method (FDTD) is widely used in computer simulations of electromagnetic phenomena. Thanks to the direct implementation of Maxwell's equations formulated in the time domain, it is especially useful for broadband simulations.
The use of the Fourier transform to analyze the simulation results in the time domain makes it possible to calculate object parameters in a wide range of frequencies on the basis of a single simulation $[12,13]$. The simulations of body shadowing presented here were performed using Remcom's XFdtd $^{\mathrm{TM}}$ software [14], which employs the FDTD method. This software enables the simulation of diverse objects segmented into cubic volumetric elements (voxels), following the method suggested by Yee [15].

The finite-difference time-domain method enables modeling of objects composed of diverse materials, which is why it is recommended in studies of the influence of electromagnetic waves on human bodies [16]. Many numerical models employing FDTD have been developed for the purpose of simulating the human body. These differ mainly in terms of the number of modeled tissues and the anatomical accuracy of the body structure [17]. The analyses presented in this paper utilize the NMR Hershey model, which is accessible in XFdtd software in several versions, with voxel sizes varying from $1 \mathrm{~mm}$ to $10 \mathrm{~mm}$.

The software performs a segmentation of the simulated object into voxels, with dimensions corresponding to the length of the electromagnetic waves which penetrate the object under analysis. Appropriate selection of the elementary cell size into which the object is divided is of primary importance for the accuracy of the calculations. A commonly applied rule is to assume the length of the side of the cell to be equal to one-tenth of the length of the shortest electromagnetic wave analyzed in the simulations $\lambda_{\text {min }}$ :

$$
\lambda_{\min }=\frac{c_{0}}{f_{c} \cdot \sqrt{\mu_{r} \varepsilon_{r}}}
$$

where $\lambda_{\min }$ is the length of the shortest wave in the model, $c_{0}$ is the speed of light in a vacuum, $f_{c}$ is wave frequency, $\mu_{r}$ is the maximum relative magnetic permeability of the model object, and $\varepsilon_{r}$ is the maximum relative electric permittivity of the model object.

The need to model the entire simulation domain using voxels with side lengths that are a faction of the wavelength in size limits the usability of this method for problems that are of a physical size comparable to tens or at least hundreds of the wavelength. This is due to the fact that the computer memory needed for simulations has to be large enough to store the data describing the material properties and electromagnetic field parameters for all the voxels in the simulation domain. For simulations that include human body models, the effect of shortening the wave in the dielectric makes the voxel size very small. For an average human body, the relative electric permittivity of the model material is $\varepsilon=38.5$, whereas its specific conductance is $\sigma=2.4 \mathrm{~S} / \mathrm{m}$. These values are typical for models used in tests of cellular system terminals [18] and can be used to approximate the average wavelength when it passes through a human body. At a frequency of $3.6 \mathrm{GHz}$, the average wavelength in a human body according to (1) is $\lambda_{\min }=13.4 \mathrm{~mm}$. The average voxel size should then be $1.34 \mathrm{~mm}$. The amount of computer memory needed to model only the volume of the body of an adult man, $1.8 \mathrm{~m}$ in height, at $3.6 \mathrm{GHz}$ is $5.8 \mathrm{~GB}$. 


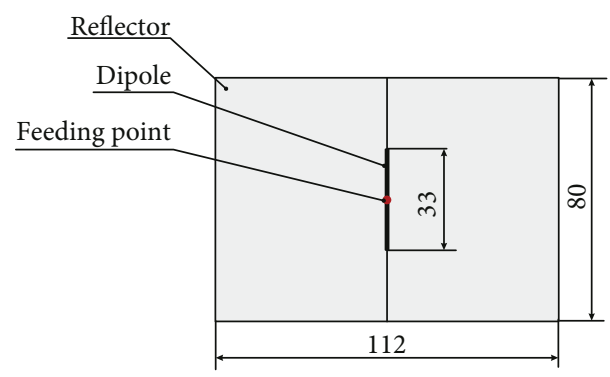

(a)

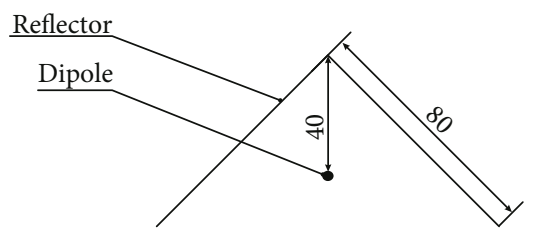

(b)

FIGURE 5: Dimensions of the corner reflector antenna used for simulations using the FDTD method (given in $\mathrm{mm}$ ): (a) front view; (b) top view.

XFdtd software makes it possible to segment the simulated object into elements with identical dimensions as well as to increase the local grid density for selected spatial variable ranges ( $x, y$, and $z$ in the Cartesian coordinate system). Hence, it is possible to adjust the voxel size to the length of the wave in a particular object. In the simulations made for this study, the voxel size for the body model was $1.34 \mathrm{~mm}$ and for the air volume $8 \mathrm{~mm}$. The antennas were modeled with $0.5 \mathrm{~mm}$ voxels, which are suitable for thin wires. To model the geometry of the measurement experiment, the amount of computer memory required was $11 \mathrm{~GB}$. This allowed the simulations to be run on a computer equipped with two Nvidia Tesla 2070 GPU cards with multicore processors (448 cores) capable of running massively parallel computations and with $6 \mathrm{~GB}$ of memory each. The simulation time was $70 \mathrm{~min}$.

The Rohde \& Schwarz HF907 antennas that were used in the measurements have a complex structure (see Figure 3), which enable them to achieve wide bandwidth operation covering a frequency range from $0.8 \mathrm{GHz}$ to $18 \mathrm{GHz}$. Due to the complex shape of the inner elements inserted into the horn, it was not possible to measure precisely the dimensions of the hidden parts, as would be necessary to model this antenna in XFdtd software. Instead, a simple corner reflector antenna was modeled for the $3.6 \mathrm{GHz}$ frequency, which has very similar electric parameters to the broadband horn antenna. The geometry of the corner reflector antenna is presented in Figure 5.

Both antennas have a directional radiation pattern and linear polarization (vertical in this experiment). They exhibit a gain of $9.5 \mathrm{dBi}$ in the frequency of interest and good impedance matching (the VSWR of the horn antenna is 1.3 and that of the corner reflector antenna 1.45). A comparison of the radiation patterns of each antenna is provided in Figure 6, where the gain values for linear, vertical polarization are shown, normalized to maximum values. For beamwidths of $90^{\circ}$ in the vertical plane and of $60^{\circ}$ in the horizontal plane, the difference between the gains of the two antennas is less than $1 \mathrm{~dB}$. The beamwidth required for exposure of the whole body in the experimental setup to antenna radiation is $98^{\circ}$ in the vertical plane and $32^{\circ}$ in the horizontal plane (for body orientation number 1, according to Figure 2). Because both the horn antenna and the corner reflector antenna have similar radiation patterns in this range of angles, it was assumed that the reflector antenna could be used in the XFdtd program to model radiation from the measurement antenna.

The measurement experiment was performed in an anechoic chamber with absorbers placed on the floor. To simulate this environment, the absorbing boundary condition was applied to simulations in XFdtd on each border of the simulation domain. The soles of the feet of the human body model were placed on the absorbing boundary.

The first stage of the numerical experiment conducted with XFdtd was performed with two corner reflector antennas, which were placed in the same positions relative to the body model as the horn antennas during the measurements. The path loss obtained at $3.6 \mathrm{GHz}$ for body orientation 1 was $44 \mathrm{~dB}$, and for body orientation 2 , it was $51 \mathrm{~dB}$. These values are very similar to those obtained in the measurement experiment, proving the adequacy of the model antenna.

The interaction of a human body with electromagnetic waves results in a complex spatial distribution of fields, depending on the distances involved and the orientation of the body towards the incident wave. Using the FDTD computer simulation method and with a heterogeneous model of the human body, it is possible to study the body shadowing effect with various distances between the body and the antenna. Simulations were made of the electric field spatial distribution around a human body placed in the line of the beam from a transmitting reflector antenna. In Figure 7, the distribution of the electric field is presented for body orientation 1, while Figure 8 presents the same results for the body orientation 2 . In the shadow region on the opposite side of the body to the transmitting antenna, the electric field intensity is significantly lower than that on the side of the transmitting antenna. The shadow region differs in terms of field intensity and spatial distribution depending on the spatial orientation of the body.

Another important factor influencing field intensity in the shadow region is distance from the body. To analyze this effect, simulations were made for different distances between the body and the receiving antenna. A reflector antenna was used for signal transmission, and a shortened half-wave dipole antenna in the vertical position was used for signal reception. This kind of receiving antenna was used because of its simple design and the possibility of placing it closer to the body than would be possible with a larger corner reflector antenna. A dipole antenna was also modeled, using a lower number of small voxels compared to the simulations of the corner reflector antenna $(0.5 \mathrm{~mm}$, suitable for modeling the thin dipole wire). This enabled simulation, within the available computational resources, of an antenna positioned at a distance of $1.7 \mathrm{~m}$ from the centre of the human body in body orientation 1 . In the case of body orientation 2 , it was possible to extend the distance to $2.1 \mathrm{~m}$ with the dipole 


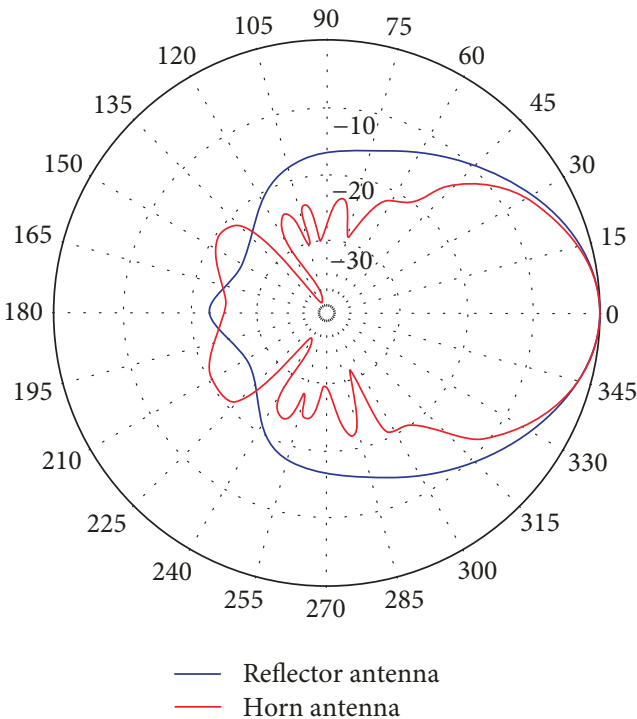

(a)

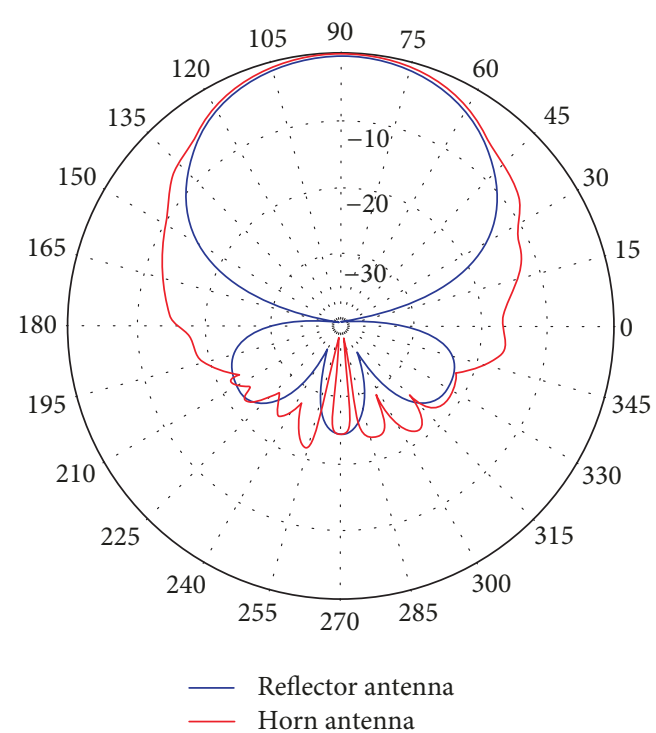

(b)

FIGURE 6: Radiation pattern of horn antenna (measured) and corner reflector antenna (simulated in XFdtd), normalized to a maximum value of $9.5 \mathrm{dBi}$ with vertical polarization: (a) horizontal plane; (b) vertical plane.
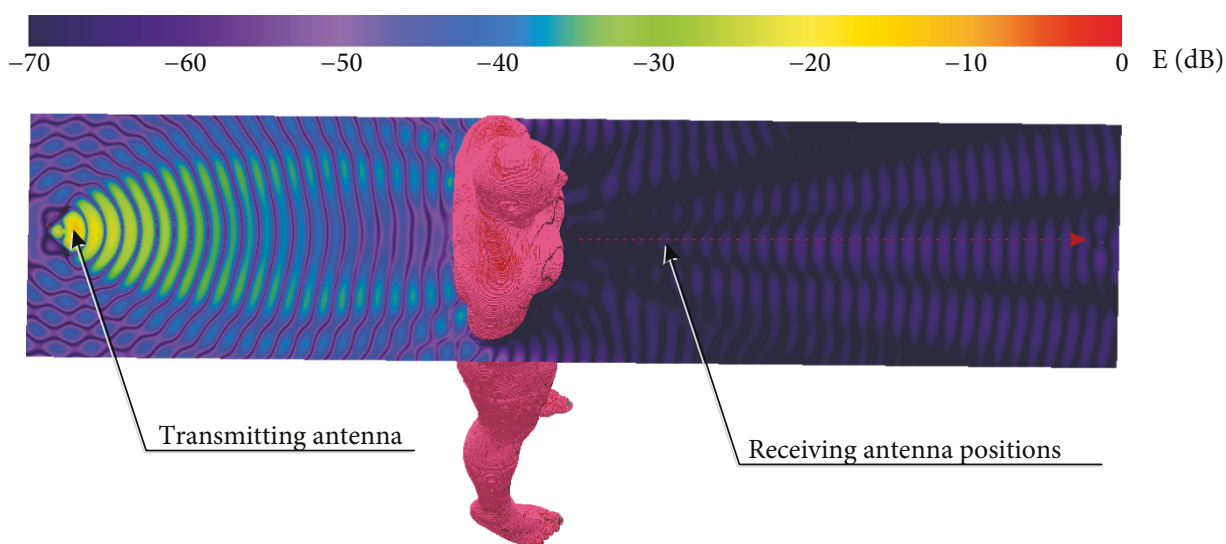

FIGURE 7: Simulation results for electric field strength obtained using FDTD—body orientation 1.

\begin{tabular}{lllllllll}
\hline-70 & -60 & -50 & -40 & -30 & -20 & -10 & & $\mathrm{E}(\mathrm{dB})$
\end{tabular}

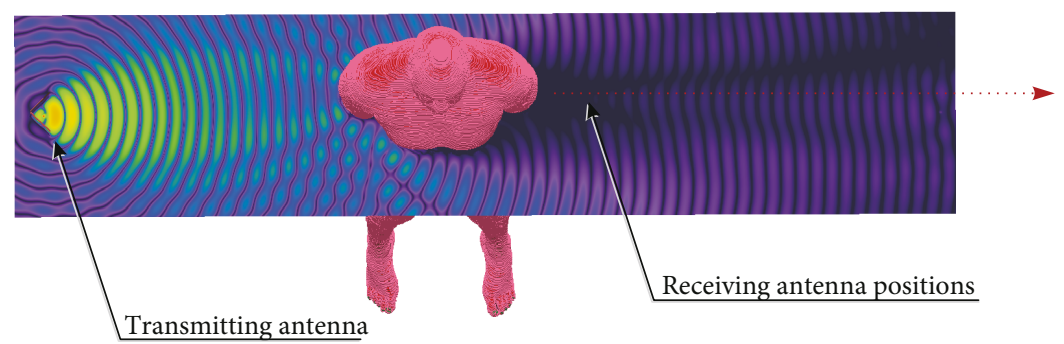

FIGURE 8: Simulation results for electric field strength obtained using FDTD_body orientation 2.

probe. Figures 7 and 8 show the positions of the dipole receiving antennas.

The results of the path loss simulations with a reflector transmitting antenna and dipole receiving antenna are presented in Figure 9. The measurements and simulations for the two reflector antennas were performed with a reference distance of $1.13 \mathrm{~m}$ from the body. This point is marked by a red dotted line in Figure 9. At this point, the path loss for body orientation 1 was $51.5 \mathrm{~dB}$, and for body orientation 2, it was $58.5 \mathrm{~dB}$. These values are $7.5 \mathrm{~dB}$ higher than those 


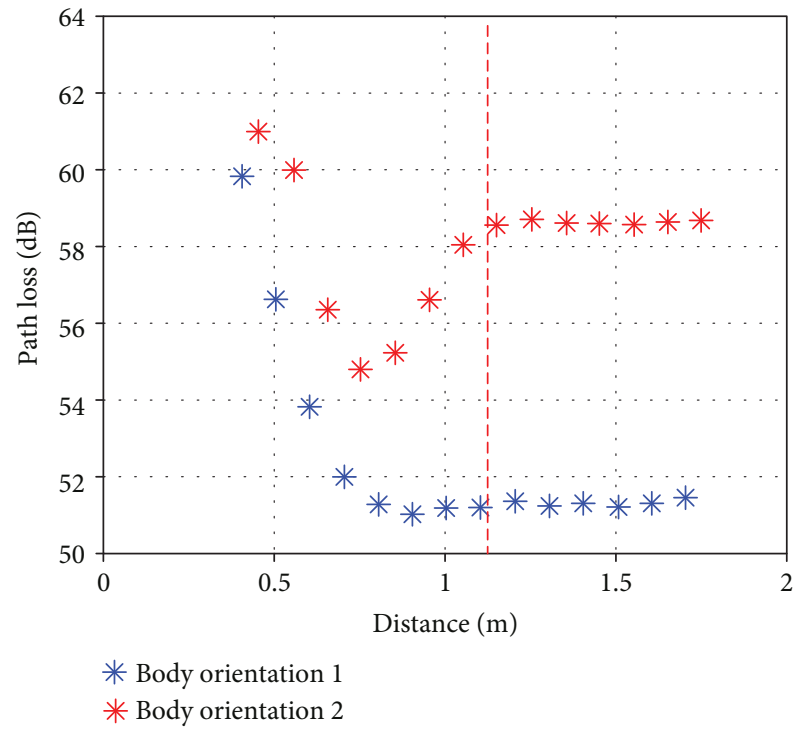

FIGURe 9: Path loss obtained using FDTD for a dipole receiving antenna at various distances from the body.

obtained in the simulations with two reflector antennas because the gain of the dipole is approximately $7.5 \mathrm{~dB}$ lower than that of the reflector antenna.

\section{Results and Discussion}

3.1. Simulations of the Body Shadowing Effect Using the Unified Theory of Diffraction. Simulations of the body shadowing effect using the FDTD method have limited applicability for the design of wireless systems. The very high demand for computer resources limits the physical size of the geometry that surrounds the body model in simulations. For the frequency of interest, $3.6 \mathrm{GHz}$, it was possible to simulate path loss for distances of only a few meters from the human body. This is too small a region to model the path loss in modern wireless systems, which operate at much larger distances, from few tens of meters to kilometers. Ray-based numerical methods are free from this limitation $[19,20]$. They are capable of simulating interactions between large objects, even for very short waves, using the resources of a typical personal computer. This motivated the elaboration of a model of the human body for use in simulations of the shadowing effect using software applying ray-based methods for wireless system design.

The Remcom XGtd program was used for elaboration of the human body model [21]. This is a general purpose raybased electromagnetic analysis tool. Its capabilities include modeling the propagation of electromagnetic waves in the vicinity of complex objects (such as aircraft, vehicles, and anechoic chambers), performing antenna placement analysis, and determining the influence of objects on the far zone patterns of antennas. XGtd models the physical characteristics of objects and anechoic chambers, performs electromagnetic calculations, and then evaluates the signal propagation characteristics. It can be used to predict how the transmitters and receivers interact with large objects, calculating the signal strength, path loss, and far zone antenna gain.
In the XGtd program, the simulations are made by shooting rays from the transmitters and propagating them through the defined geometry of the modeled object. The rays interact with the geometrical features of the objects on their way to the receiver. Ray interactions that are simulated include reflections from feature faces, diffraction around feature edges, and transmission through feature faces. The raybased solvers use the uniform theory of diffraction (UTD) to evaluate the electric field along the ray path [22-24]. UTD provides accurate results when the scenario geometry is large compared to the wavelength of the propagating wave. For typical applications, UTD-based models provide accurate predictions from approximately $100 \mathrm{MHz}$ to approximately $100 \mathrm{GHz}$. The X3D propagation model applied in XGtd includes atmospheric absorption, extending the validity of its wave propagation calculations up to millimeter wave frequencies. In each receiver location, contributions from arriving ray paths are combined and evaluated to determine predicted quantities such as electric and magnetic field strength, received power, interference measures, path loss, delay spread, direction of arrival, impulse response, electric field versus time, electric field versus frequency, and the power delay profile.

UTD is applicable for simulating the interaction of electromagnetic waves with objects of the size significantly greater than the wavelength. In the considered case of body shadowing at $3.6 \mathrm{GHz}$, this condition is satisfied because the height of the human body is approximately 20 times greater than the wavelength. The radii of the cylinders approximating the human body should be also significantly greater than the wavelength. For the considered frequency, the crosssectional diameter is only 1.8 times greater than the wavelength, which is at the limit of the UTD applicability range.

Despite the limitations outlined above, the UTD method was utilized to model the body shadowing effect in a $2.4 \mathrm{GHz}$ band [3]. The authors considered different elementary objects like rectangular blade, parallelepiped, and cylinder to model the human body. It was possible to estimate only the mean attenuation introduced by the body, averaged over different placements of a person towards the transmitting antenna. These models do not take into account the orientation of the human body towards the ray. This may limit the accuracy of simulations obtained with such model in the case of systems operating in multipath environment, where the rays that are interacting with the human body may hit it from different angles. As shown above, the attenuation introduced by the body may differ between different body orientations up to $8 \mathrm{~dB}$ (see Figure 9).

The model of the human body proposed here for use in the XGtd program is capable of simulating the body shadowing effect with respect to different orientations of the human body towards the ray. Simulation of the body shadowing effect in XGtd required a simplified model of a human body. For this purpose, a cylindrical model was developed that approximates the shape of the body using cylindrical objects. The cylindrical model of the body was originally created by the author for simulations using the FDTD method $[25,26]$ and for measurement experiments. It was shown in [25] with measurement results that this geometry gives a good 


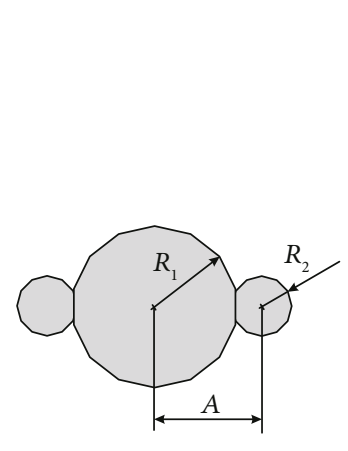

(a)

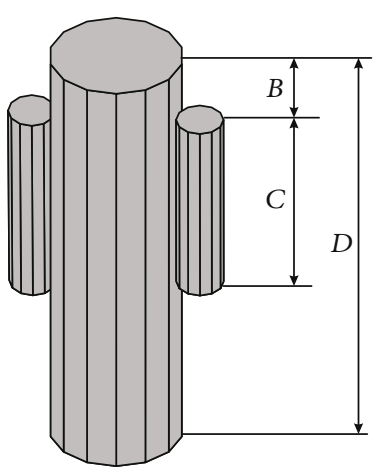

(b)
FIGURE 10: Geometry of the simplified human body model: (a) top view; (b) side view.

approximation of the human body in standing position for body shadowing analysis. In the present study, the simplest version of the model was used, consisting of 3 cylinders. The largest cylinder covers the volume of the head, trunk, and legs. Two smaller cylinders model the arms. The geometry of the model is presented in Figure 10. The dimensions that were taken from the model that was successfully used in measurements [25] are as follows: $A=0.2 \mathrm{~m}, R_{1}=0.1575$ $\mathrm{m}, R_{2}=0.055 \mathrm{~m}, B=0.195 \mathrm{~m}, C=0.53 \mathrm{~m}$, and $D=1.8 \mathrm{~m}$.

The numerical experiment conducted in XGtd was based on the same geometric setup as the experiment made with XFdtd software. The transmitting antenna also had the same radiation pattern and gain as the reflector antenna used for the simulation in the XFdtd program. It was possible to import the radiation pattern of this antenna simulated in XFdtd to XGtd using the UAN file. The receiving antenna was again a half-wave dipole. The transmitting antenna was placed $1.13 \mathrm{~m}$ from the body and $1.3 \mathrm{~m}$ from the floor. The polarization of the wave was linear in the vertical plane.

As presented above, the initial measurement experiment was performed in an anechoic chamber with absorbers placed on the floor. The corresponding numerical experiment conducted in XFdtd also assumed no interaction between the wave and the floor because the absorbing boundary condition was defined at the level of the base of the human body. For the purpose of the simulations in XGtd, it was also assumed that there would be no interaction between the electromagnetic wave and either the floor or the base of the body. To achieve this, a model of the anechoic chamber was used in the XGtd simulation, surrounding the human body and the transmitting and receiving antennas. The anechoic chamber consisted of perfectly absorbing material. The dimensions of the chamber model were as follows: the width and depth $6 \mathrm{~m}$ and height $2.5 \mathrm{~m}$.

The bottom floor of the chamber was at the same level as the base of the human body model. The material used to model the human body had the same properties as the simplified human body model used for the FDTD simulations $[25,26]$. Its relative electric permittivity was $\varepsilon=52$, and its specific conductance was $\sigma=1.8 \mathrm{~S} / \mathrm{m}$.

In the XGtd program, the cylinders are approximated as regular polygonal prisms with different numbers of side

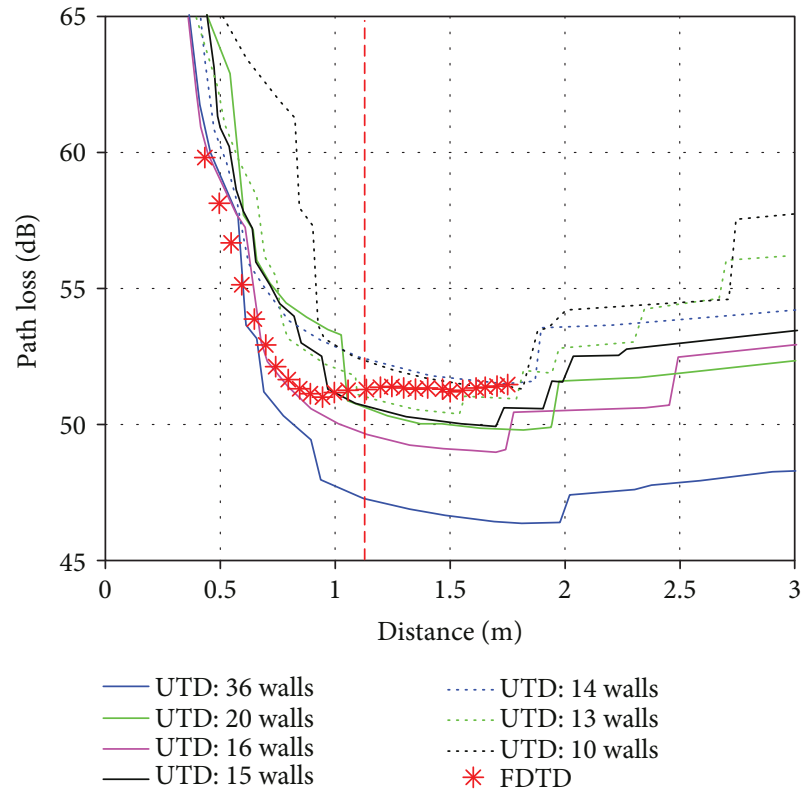

Figure 11: Path loss results obtained in simulations using XGtd (UTD method) and XFdtd (FDTD method) for body orientation 1 and for different numbers of side walls in the UTD model.

walls. The number of side walls utilized to approximate the cylindrical model of the human body had to be set up experimentally. For this reason, the simulations of path loss were performed with different numbers of faces (side walls) that approximated the cylinders. The number of polygonal prism faces examined here was in the range of 10-36. The number of side walls of arm cylinders and trunk cylinders was investigated, but it appeared that the model was more sensitive to the number of walls used in the trunk element and less sensitive to the approximation of arms. After making a number of simulations, the best approximation of the arm cylinders was found to be 12 side walls.

The influence of the number of sidewalls used in the trunk cylinder on the path loss simulations is presented in Figures 11 and 12, comparing the results obtained for the same geometry in XFdtd, utilizing FDTD and the heterogeneous body model. In Figure 11, the simulations were performed for the first orientation of the body towards the transmitting antenna (antenna directed towards the back of the body). In Figure 12, the second orientation was examined (antenna is directed towards the arm).

To identify the variant of the model that gives the results similar to FDTD simulations, the analysis of median values of path loss was performed. In Table 1 , the median values are presented for the first body orientation while Table 2 presents results for the second orientation of the body. Median values of path loss were calculated for the distances where the body shadowing effect has the greatest influence to the path loss. Two ranges of distances from the body were considered: $0.5 \mathrm{~m}-1 \mathrm{~m}$ and $1 \mathrm{~m}-1.5 \mathrm{~m}$. In Tables 1 and 2 , the results obtained with FDTD are the reference for the results given by UTD models. In columns $\mathrm{c}$ and $\mathrm{e}$, the difference between the median values obtained from both methods are calculated. 


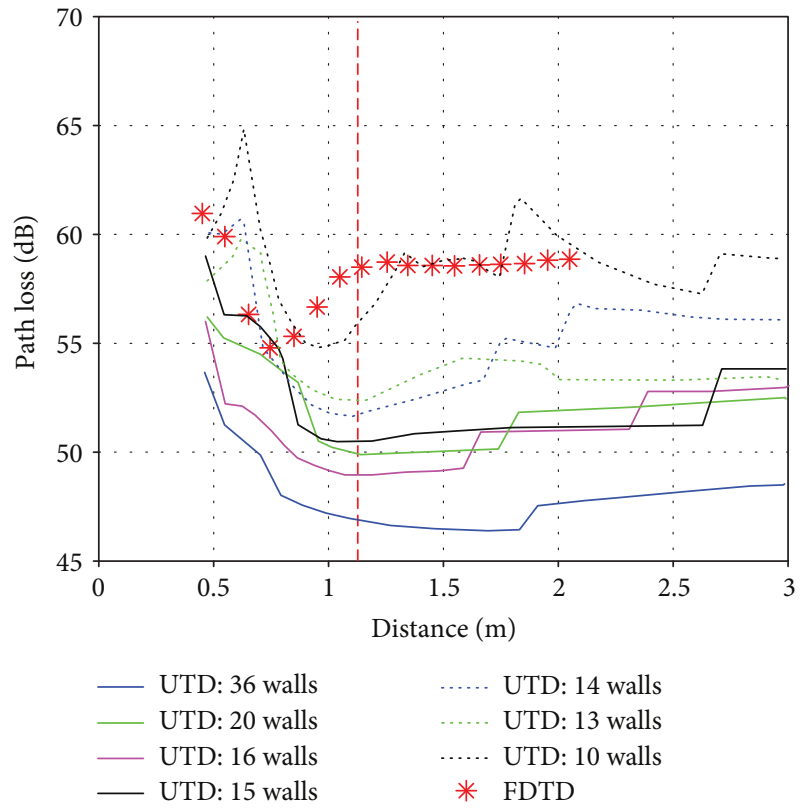

FIGURe 12: Path loss results obtained using XGtd (UTD method) and XFdtd (FDTD method) for body orientation 2 and for different numbers of side walls in the UTD model.

The best results were obtained for the approximation of the main cylinder with 14 sides and arm cylinders with 12 sides (as presented in Figure 10). In this case, there are similar differences between FDTD and UTD simulations for both spatial orientations of the body and transmitting antenna. The average difference of median values obtained with this model and FDTD in two considered range of distances and two spatial configurations is $2.9 \mathrm{~dB}$. For instance, in the case of the model where the main cylinder contains 10 side walls, there is a good correspondence to FDTD only in the second geometrical case (average difference is $1.2 \mathrm{~dB}$ ), while in the first body orientation, it shows less correspondence to FDTD (average difference is $5.4 \mathrm{~dB}$ ) than the 14-wall model.

The model with 14 side walls on the trunk was also verified in the reference point, where the measurements and FDTD simulations were compared. In Figure 11, the reference distance for the dipole antenna at $1.13 \mathrm{~m}$ from the centre of the body is marked with a red dotted line. At this distance, the path loss calculated by the XFdtd program was $51.5 \mathrm{~dB}$, while the value obtained in XGtd was $52.5 \mathrm{~dB}$. At shorter distances from the body, the correspondence between the results obtained using the two methods was not worse than $3.5 \mathrm{~dB}$. For the longest distance that could be simulated with XFdtd $(1.7 \mathrm{~m})$, the methods gave almost the same results.

The results for the second body alignment are shown in Figure 12. In this case, the FDTD-based path loss for the reference point was $58.5 \mathrm{~dB}$ and that for UTD was $52 \mathrm{~dB}$. The two methods gave very similar results for shorter distances, while for maximum distances from the body, there were differences of $3 \mathrm{~dB}$ between the simulations.

The CPU time needed to run the simulations in XGtd was 5 min using the same computer that was used for the simulations in XFdtd.
3.2. Simulations of the Wireless System Performance including Body Shadowing Effect with the Simplified Human Body Model. The simplified body model that was proposed in this paper for body shadowing simulations can be applied for the analysis of wireless system performance in the presence of people. To show the potential application of this model, a case study was performed. It presents the influence of the presence of people on the system performance that was obtained with the UTD-based model of the human body.

The example presented below covers the simulation of path loss in a $5 \mathrm{G}$ network operating in the $3.6 \mathrm{GHz}$ band. It was assumed that the system was designed to operate in the sport stadium where the number of people and their spatial density can vary significantly. In such large objects compared to the wavelength (in this case, the wavelength is $8.3 \mathrm{~cm}$ ), only the ray-based electromagnetic analysis tool can be used. In this case, the XGtd program was applied together with the 14-wall simplified human body model to analyze the path loss. For simplicity of presentation, only a small section of the stadium was considered here. In Figure 13, the section of the stadium $5 \mathrm{~m}$ by $4 \mathrm{~m}$ large is presented. In the considered case shown in Figure 13(a), there are 5 people placed in one row while in the case in Figure 13(b), 10 people occupy 2 parallel rows. The rows are elevated from each other by the level of $30 \mathrm{~cm}$. In this simple case study, it was assumed that the transmitter would be placed 5 meters in front of the people. The path loss was simulated between one transmitter and the number of receivers that were located behind the row of spectators. This corresponds to the analysis that should be performed for the system coverage in the upper places of the stadium section. The receivers are distributed along the line of length equal to $2.5 \mathrm{~m}$ that was placed $1 \mathrm{~m}$ from the back of the people in the front row (blue dotted line in Figure 13). It was assumed that half-wave dipoles would be used in both the transmitter and receivers. The polarization of the wave was linear in the vertical plane.

Figure 14 presents the path loss simulated for the receivers located along the line on the back of the people. The results were obtained for 3 cases: where no people were present, only 5 people were placed in one row (see Figure 13(a)), and 10 people were placed in 2 rows (see Figure 13(b)). In the first case, the path loss is almost constant along the receivers' line and equal to $59 \mathrm{~dB}$. The case for which 5 people were placed in one row shows the increase of the path loss median value to $61.4 \mathrm{~dB}$ and a significant increase in path loss variance. For this case, standard deviation of the path loss was equal to $17 \mathrm{~dB}$. With 10 people placed in 2 rows, the median value of path loss increased to $64.3 \mathrm{~dB}$ and standard deviation of the path loss was equal to $7.7 \mathrm{~dB}$.

This case study shows the influence of the people's presence on the path loss in the wireless system that operates in $3.6 \mathrm{GHz}$. The difference in median value of path loss caused by the presence of people can reach $5 \mathrm{~dB}$. The local differences in path loss can be higher than $20 \mathrm{~dB}$.

\section{Conclusions}

This study sets out to investigate the effect of shadowing caused by obstruction of transmission paths in wireless 
TABLE 1: Median values of path loss obtained for different geometries of models-body orientation 1.

\begin{tabular}{|c|c|c|c|c|}
\hline $\begin{array}{l}\text { Human body model } \\
\text { a }\end{array}$ & $\begin{array}{c}\text { Median of path loss } \\
\text { in the distance from } \\
0.5 \mathrm{~m} \text { to } 1 \mathrm{~m}(\mathrm{~dB}) \\
\mathrm{b}\end{array}$ & $\begin{array}{l}\text { Difference to FDTD } \\
\text { in the distance from } \\
0.5 \mathrm{~m} \text { to } 1 \mathrm{~m}(\mathrm{~dB}) \\
\mathrm{c}\end{array}$ & $\begin{array}{c}\text { Median of path loss } \\
\text { in the distance from } \\
1 \mathrm{~m} \text { to } 1.5 \mathrm{~m}(\mathrm{~dB}) \\
\mathrm{d}\end{array}$ & $\begin{array}{l}\text { Difference to FDTD } \\
\text { in the distance from } \\
1 \mathrm{~m} \text { to } 1.5 \mathrm{~m}(\mathrm{~dB}) \\
\mathrm{e}\end{array}$ \\
\hline FDTD: heterogeneous & 51.8 & - & 51.3 & - \\
\hline UTD: 36 walls & 50.6 & 1.2 & 47 & 4.3 \\
\hline UTD: 20 walls & 54.8 & 3 & 50.2 & 1 \\
\hline UTD: 16 walls & 51.8 & 0.03 & 49.3 & 1.9 \\
\hline UTD: 15 walls & 54.8 & 2.9 & 50.4 & 0.8 \\
\hline UTD: 14 walls & 54.2 & 2.4 & 52 & 0.7 \\
\hline UTD: 13 walls & 54.6 & 2.7 & 50.7 & 0.6 \\
\hline UTD: 10 walls & 61.9 & 10 & 51.9 & 0.7 \\
\hline
\end{tabular}

TABle 2: Median values of path loss obtained for different geometries of models-body orientation 2.

\begin{tabular}{|c|c|c|c|c|}
\hline $\begin{array}{l}\text { Human body model } \\
\text { a }\end{array}$ & $\begin{array}{c}\text { Median of path loss } \\
\text { in the distance from } \\
0.5 \mathrm{~m} \text { to } 1 \mathrm{~m}(\mathrm{~dB}) \\
\mathrm{b}\end{array}$ & $\begin{array}{c}\text { Difference to FDTD } \\
\text { in the distance from } \\
0.5 \mathrm{~m} \text { to } 1 \mathrm{~m}(\mathrm{~dB}) \\
\mathrm{c}\end{array}$ & $\begin{array}{l}\text { Median of path loss } \\
\text { in the distance from } \\
1 \mathrm{~m} \text { to } 1.5 \mathrm{~m}(\mathrm{~dB}) \\
\mathrm{d}\end{array}$ & $\begin{array}{l}\text { Difference to FDTD } \\
\text { in the distance from } \\
1 \mathrm{~m} \text { to } 1.5 \mathrm{~m}(\mathrm{~dB}) \\
\mathrm{e}\end{array}$ \\
\hline FDTD: heterogeneous & 56.5 & - & 58.6 & - \\
\hline UTD: 36 walls & 48.9 & 7.6 & 46.7 & 11.9 \\
\hline UTD: 20 walls & 54.1 & 2.4 & 49.9 & 8.7 \\
\hline UTD: 16 walls & 50.9 & 5.6 & 49 & 9.6 \\
\hline UTD: 15 walls & 55.1 & 1.4 & 50.6 & 8 \\
\hline UTD: 14 walls & 54.2 & 2.3 & 52.1 & 6.5 \\
\hline UTD: 13 walls & 54.8 & 1.7 & 52.8 & 5.8 \\
\hline UTD: 10 walls & 58.1 & 1.6 & 57.6 & 0.9 \\
\hline
\end{tabular}

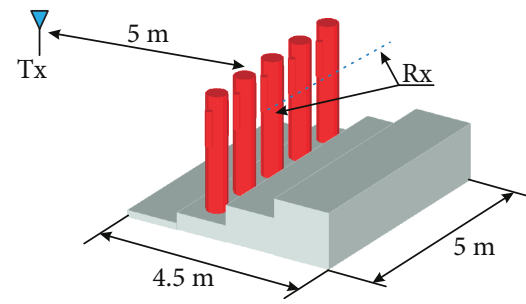

(a)

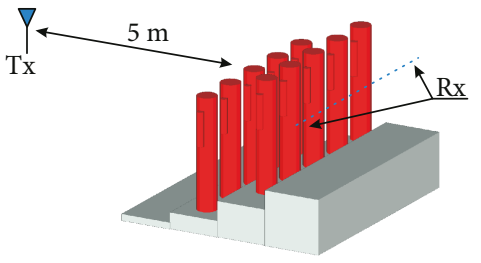

(b)

Figure 13: The simulation scenarios of the 5G system: (a) 5 people in one row; (b) 10 people in 2 rows.

systems by a human subject. The $3.6 \mathrm{GHz}$ frequency was used, which is one of the bands being considered for $5 \mathrm{G}$ systems. The measurement results were used as the reference for computer simulations made with a full-wave FDTD-based program (XFdtd) with a heterogeneous human body model. The results obtained from the simulations for the reference point were very similar to the measurements from the two body orientations towards the transmitting and receiving antennas. This showed the validity of the FDTD model for simulating human body shadowing. Changes in path loss were then examined for different distances of the receiving antenna from the body. Due to the high computational demands of FDTD code and the limited computer resources available, it was possible to examine only very limited distances with this method, up to $2.1 \mathrm{~m}$, depending on the body orientation. The results were then used as the reference for experiments made in a UTD-based computer program.

Ray-based computer programs are widely used in the design of wireless systems operating in urban or indoor environments. They are capable of simulating electromagnetic effects at much larger distances from the transmitter than full-wave methods (such as FDTD). To simulate the body shadowing effect in a ray-based computer program, a simplified human body model was proposed. This approximates a body using polygonal prisms made from a dielectric material. The results obtained using this model corresponded to the data obtained with the full-wave FDTD method. For the first orientation of the human body towards the transmitter, the differences in path loss did not exceed $3 \mathrm{~dB}$. For the second orientation, the difference 


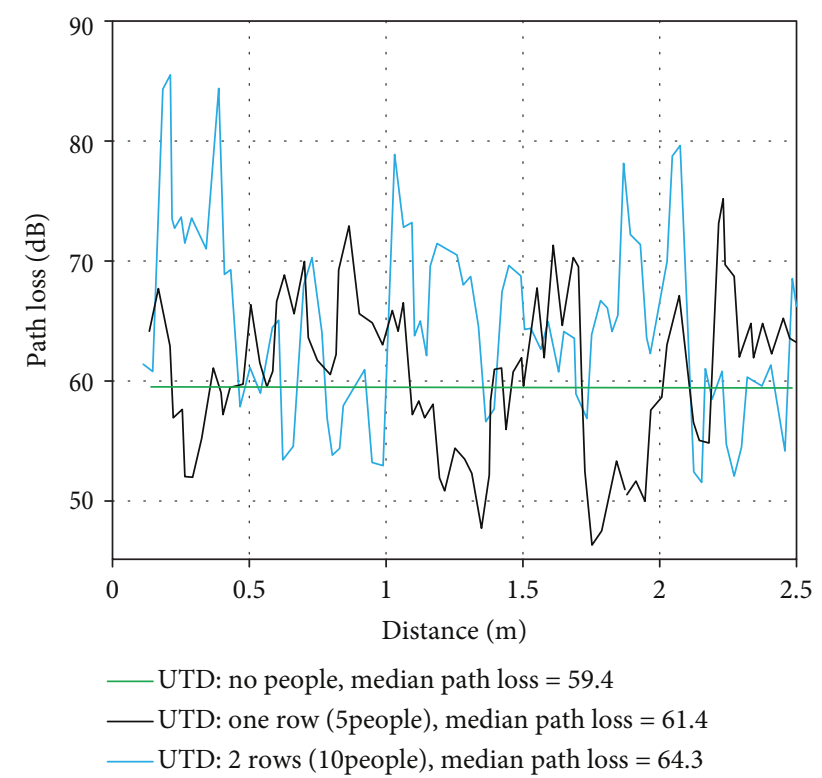

FIGURE 14: The results of path loss simulation for the case where there were no people, 5 people in one row, and 10 people in 2 rows.

did not exceed $7 \mathrm{~dB}$ in the worst case, but for distances between the antenna and the body that exceeded $2 \mathrm{~m}$, the difference was not greater than $3 \mathrm{~dB}$.

The proposed model could be used for simulations of the influence of human bodies on wireless systems performed with ray-based programs. It is capable of simulating the body shadowing effect with respect to different orientations of the human body towards the ray. This is very relevant for analysis of the wireless coverage of $5 \mathrm{G}$ systems in multipath environment like the interiors of buildings. The small computational cost (5 minutes) of simulations performed with the proposed model of the human body makes such analysis possible even for large interiors.

The case study that was presented in the paper was performed with the proposed human body model. It shows the influence of the people's presence on the path loss in the wireless system that operates in $3.6 \mathrm{GHz}$. In the considered case, the difference in median value of path loss caused by the presence of people can reach $5 \mathrm{~dB}$. The local differences in path loss can be higher than $20 \mathrm{~dB}$. This justifies the need of simulation of human body shadowing on the system performance also in large objects.

The scope of the paper covers only the $3.6 \mathrm{GHz}$ band. Further research will be carried out to investigate also other bands $(2.1 \mathrm{GHz}, 2.4 \mathrm{GHz}$, and $5.8 \mathrm{GHz})$ that are widely utilized in wireless sensor networks. Also, the analysis of other shapes of models with special attention to elliptical cylinders applied to human body modeling will be the subject of further investigation.

\section{Data Availability}

The data used to support the findings of this study are available from the corresponding author upon request.

\section{Conflicts of Interest}

The author declares that there is no conflict of interest regarding the publication of this paper.

\section{Acknowledgments}

The research being reported in this publication was funded by Lodz University of Technology, Institute of Electronics, within the frames of an internal research grant.

\section{References}

[1] S. Mojaiana, "Impacts of human body shadowing in wireless body-centric communications," International Journal of Future Generation Communication and Networking, vol. 9, no. 6, pp. 191-200, 2016.

[2] Y. Wang, I. B. Bonev, J. O. Nielsen, I. Z. Kovacs, and G. F. Pedersen, "Characterization of the indoor multi-antenna body-to-body radio channel," IEEE Transactions on Antennas and Propagation, vol. 57, no. 4, pp. 972-979, 2009.

[3] A. D. C. de Queiroz and L. C. Trintinália, "An analysis of human body shadowing models for ray-tracing radio channel characterization," in 2015 SBMO/IEEE MTT-S International Microwave and Optoelectronics Conference (IMOC), pp. 1-5, Porto de Galinhas, PE, Brazil, 2015.

[4] S. L. Cotton, "Human body shadowing in cellular device-todevice communications: channel modeling using the shadowed $\kappa-\mu$ fading model," IEEE Journal on Selected Areas in Communications, vol. 33, no. 1, pp. 111-119, 2015.

[5] M. Peter, M. Wisotzki, M. Raceala-Motoc et al., "Analyzing human body shadowing at $60 \mathrm{GHz}$ : systematic wideband MIMO measurements and modeling approaches," in 2012 6th European Conference on Antennas and Propagation (EUCAP), pp. 468-472, Prague, Czech Republic, 2012.

[6] C. Gustafson and F. Tufvesson, "Characterization of $60 \mathrm{GHz}$ shadowing by human bodies and simple phantoms," Radioengineering, vol. 21, no. 4, pp. 979-984, 2012.

[7] K. Akimoto, S. Kameda, M. Motoyoshi, and N. Suematsu, "Measurement of human body blocking at $60 \mathrm{GHz}$ for internetwork interference of mmWave WBAN," in 2017 IEEE Asia Pacific Microwave Conference (APMC), pp. 472-475, Kuala Lumpar, Malaysia, 2017.

[8] X. Chen, L. Tian, P. Tang, and J. Zhang, "Modelling of human body shadowing based on $28 \mathrm{GHz}$ indoor measurement results," in 2016 IEEE 84th Vehicular Technology Conference (VTC-Fall), pp. 1-5, Montreal, QC, Canada, 2016.

[9] J. H. Jung, J. Lee, J. H. Lee, Y. H. Kim, and S. C. Kim, "Ray-tracing-aided modeling of user-shadowing effects in indoor wireless channels," IEEE Transactions on Antennas and Propagation, vol. 62, no. 6, pp. 3412-3416, 2014.

[10] Y. Zhao, Y. Hao, A. Alomainy, and C. Parini, "UWB on-body radio channel modeling using ray theory and subband FDTD method," IEEE Transactions on Microwave Theory and Techniques, vol. 54, no. 4, pp. 1827-1835, 2006.

[11] RSPG Strategic Roadmap Towards 5G for Europe, “Opinion on spectrum related aspects for next-generation wireless systems (5G)," (RSPG16-032) November 2016, http://rspgspectrum.eu/.

[12] A. Taflove and S. C. Hagness, Computational Electrodynamics: the Finite-Difference Time-Domain Method, Artech House, Norwood, MA, USA, 2005. 
[13] K. S. Kunz and R. J. Luebbers, The Finite Difference Time Domain Method for Electromagnetics, CRC Press, 1993.

[14] XFDTD 7.3 Reference Manual, Remcom Inc., 2014.

[15] K. Yee, "Numerical solution of initial boundary value problems involving Maxwell's equations in isotropic media," IEEE Transactions on Antennas and Propagation, vol. 14, no. 3, pp. 302-307, 1966.

[16] "IEEE Recommended Practice for Measurements and Computations of Radio Frequency Electromagnetic Fields With Respect to Human Exposure to Such Fields, $100 \mathrm{kHz}-$ $300 \mathrm{GHz}$," in IEEE Std C95.3-2002 (Revision of IEEE Std C95.3-1991), pp. 1-126, January 2003.

[17] D. Andreuccetti, R. Fossi, and C. Petrucci, "An internet resource for the calculation of the dielectric properties of body tissues in the frequency range $10 \mathrm{~Hz}-100 \mathrm{GHz}$," IFAC-CNR, Florence, Italy, 1997, http://niremf.ifac.cnr.it/tissprop/.

[18] K. Fukunaga, S. Watanabe, Y. Yamanaka, H. Asou, Y. Ishi, and K. Sato, "Dielectric properties of liquid phantoms for evaluations of mobile phones," in Proceedings of EMC'04 Sendai, pp. 805-808, Sendai, Japan, June 2004, 4B3-5.

[19] F. Saez de Adana and O. Gutierrez, Practical Applications of Asymptotic Techniques in Electromagnetics, Artech House, 2010.

[20] T. M. Macnamara, Introduction to Antenna Placement and Installation, John Wiley \& Sons, Ltd, 2010.

[21] XGtd Reference Manual, Remcom Inc., 2012.

[22] P. Pathak, W. Burnside, and R. Marhefka, “A uniform GTD analysis of the diffraction of electromagnetic waves by a smooth convex surface," IEEE Transactions on Antennas and Propagation, vol. 28, no. 5, pp. 631-642, 1980.

[23] P. Pathak, N. Wang, W. Burnside, and R. Kouyoumijian, "A uniform GTD solution for the radiation from sources on a convex surface," IEEE Transactions on Antennas and Propagation, vol. 29, no. 4, pp. 609-622, 1981.

[24] R. G. Kouyoumjian and P. H. Pathak, "A uniform geometrical theory of diffraction for an edge in a perfectly conducting surface," Proceedings of the IEEE, vol. 62, no. 11, pp. 1448-1461, 1974.

[25] Ł. Januszkiewicz and S. Hausman, "Simplified human phantoms for wireless body area network modelling," in 2015 9th European Conference on Antennas and Propagation (EuCAP), pp. 1-4, Lisbon, Portugal, 2015.

[26] L. Januszkiewicz, "Simplified human body models for interference analysis in the cognitive radio for medical body area networks," in 2014 8th International Symposium on Medical Information and Communication Technology (ISMICT), pp. 1-5, Firenze, Italy, 2014. 


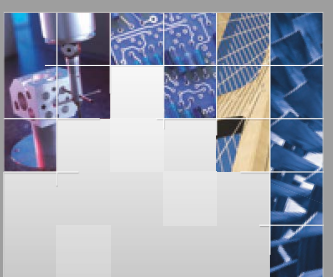

\section{Enfincering}
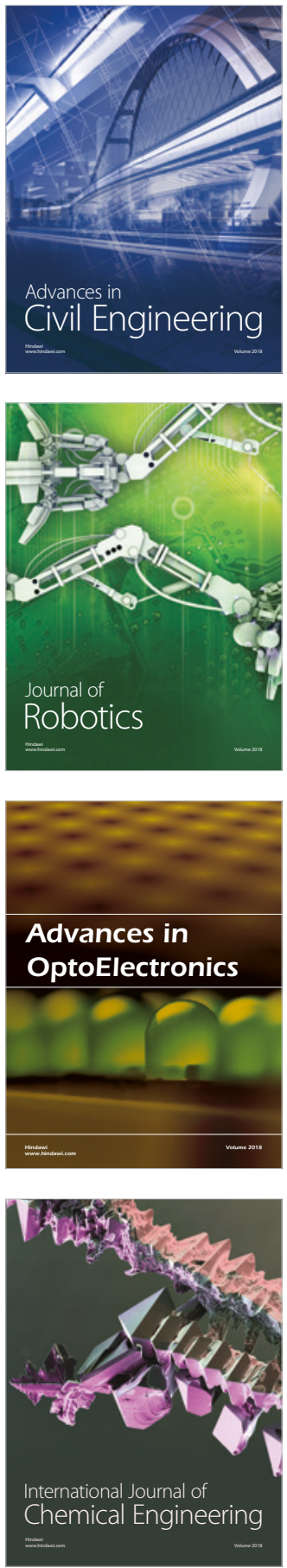

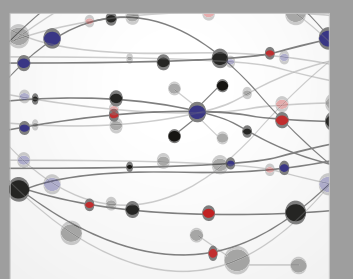

\section{Rotating \\ Machinery}

The Scientific World Journal

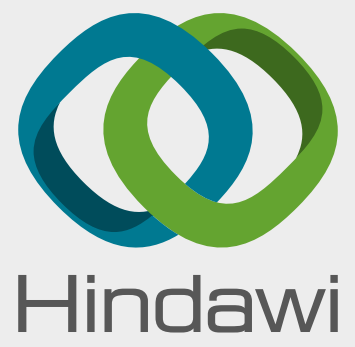

Submit your manuscripts at

www.hindawi.com
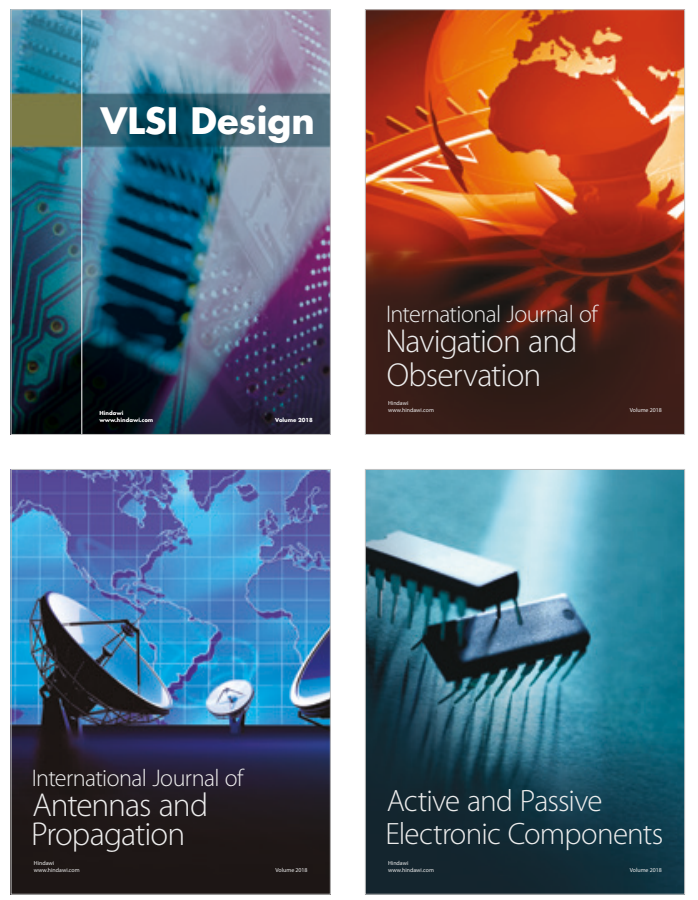
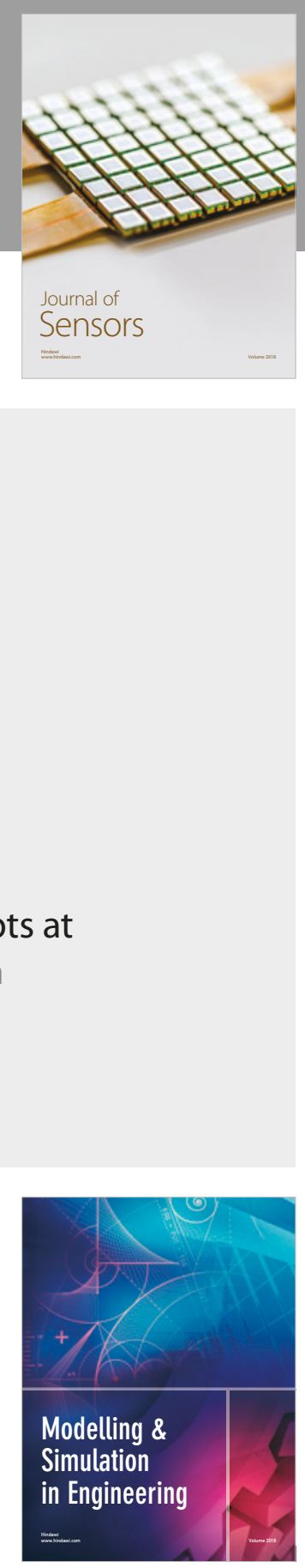

\section{Advances \\ Multimedia}
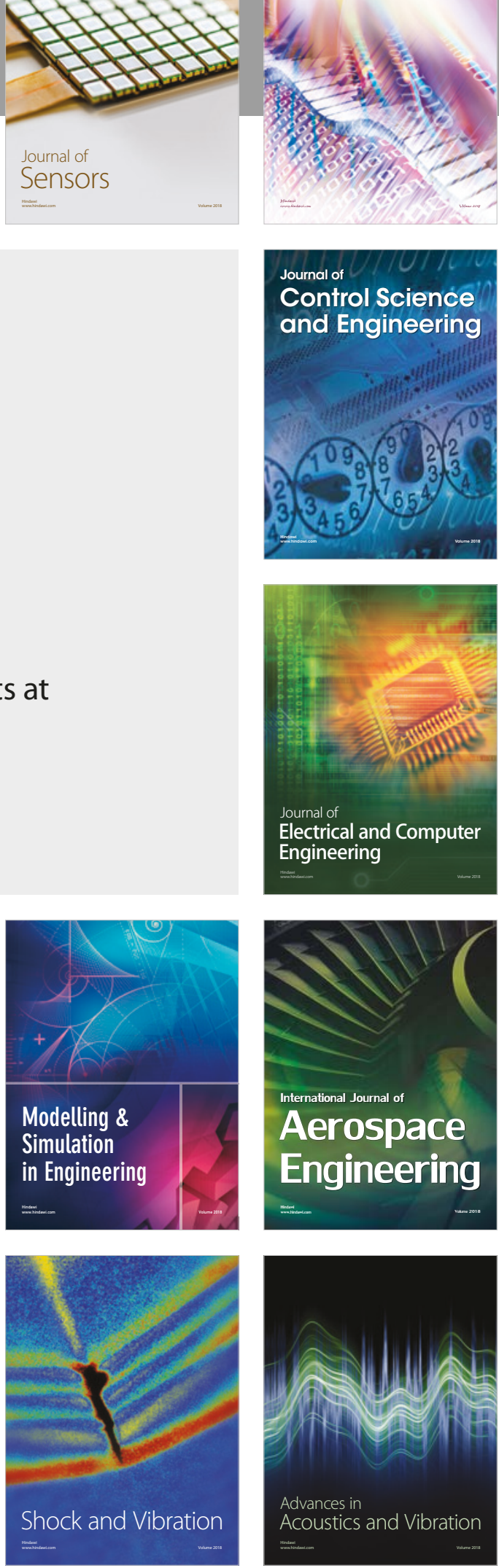